\title{
Aplicabilidad de la Teoría de la Actividad Histórico-Cultural en los estudios de comportamiento informacional
}

\author{
Raciel García Rodríguez \\ Universidad de Matanzas - UM, Cuba
}

REVIEW

\begin{abstract}
Resumen
Se expone un panorama de las potencialidades de aplicación de la Teoría de la Actividad Histórico-Cultural a los estudios de comportamiento informacional, como una herramienta holística en el empeño por lograr una mejor comprensión de los distintos modos en que los individuos buscan, encuentran, recuperan y usan la información que necesitan, desde una aproximación transdisciplinar que fortalece esta visión.
\end{abstract}

Palabras clave

Búsqueda de información; comportamiento informacional; Teoría de la Actividad Histórico-Cultural

\section{Applicability of Cultural-Historical Activity Theory on information behaviour studies}

\begin{abstract}
Potentialities on how the Cultural-Historical Activity Theory can be applied to the information behavior studies are exposed, offering an extensive batch of arguments and reasons that justify this proposal, in virtue of getting a more comprehensive understanding of the different ways through which individuals seek, search, retrieve and make use of the information they need, by also using a transdisciplinary approach that strengthens this view.
\end{abstract}

\section{Introducción}

El esfuerzo de las entidades de información por ofrecer servicios informativos más pertinentes requiere (re)conocer y estudiar más a los usuarios, no simplemente desde sus características generales o sociodemográficas (edad, sexo, nivel de escolaridad, etcétera) -como tradicionalmente se ha hecho en los estudios de usuarios- sino desde sus comportamientos informacionales previos y a formar. (Uribe, 2008)

La necesidad por indagar más profundamente en cómo las personas gestionan (buscan, hallan y usan) la información que necesitan se ha vuelto más apremiante en el marco del avance vertiginoso de la llamada Sociedad de la Información y el Conocimiento, que ha estado caracterizada por (Sánchez Díaz, 2010):

- La aparición de nuevos y diversos canales y medios de comunicación; formatos y soportes de fuentes de información; formas de acceso y manipulación; sistemas de información interactivos y participativos.

- La producción masiva de información dinámica, abierta, globalizada y ágil.

- La información como bien de consumo singular ha adquirido una dimensión estratégica. 
- La supervivencia humana, el desarrollo social y el incremento de la productividad dependen de la creación, almacenamiento, uso y distribución de información y conocimientos.

- La utilización extensiva y optimizada de las tecnologías de la información y comunicación.

Estudiar el comportamiento informacional implica ineludiblemente detenerse a valorar aquellas variables de orden sociosicológico que afectan la manera en que los seres humanos interactúan con la información.

Se ha corroborado que los estudios de comportamiento informacional han tenido una preponderancia significativa en los últimos años, dedicándose a analizar especialmente la búsqueda de información de los individuos. (Rowley \& Urquhart, 2000; Case, 2008; Rubinić, 2012)

Reafirmando esta tendencia, Izquierdo (citada por Guevara, 2004, p. 27) ha señalado que los estudios de comportamiento informacional son relevantes porque "permiten identificar las actitudes y valores de los individuos con respecto a la información en general y a las relaciones con las unidades de información en particular, así como valorar los fundamentos del desarrollo de la búsqueda, uso y diseminación de la información".

La Teoría de la Actividad Histórico-Cultural se perfila como una vía poco 'explotada' pero muy recomendable para aproximarse a los individuos en este sentido, dado el amplio espectro de análisis que presenta, a partir de la unidad básica de análisis del comportamiento de los sujetos: la actividad humana.

\section{Desarrollo}

El comportamiento informacional representa el vínculo entre la información y la condición humana; nexo en el que la información siempre ha sido un elemento esencial para la existencia del hombre, pues ella hace posible la adaptación y la supervivencia en un medio físico y social sumamente dinámico. La condición humana puede entenderse como el "conjunto de carencias informativas del hombre y las consecuencias que traen aparejadas". (Micó, 2014, p. 16)

El término en sí mismo -acuñado en la década de los años 90- ha sido objeto de discusión, pues especialistas indican que este se refiere más bien a la información que a los individuos, cuando en realidad son los individuos quienes tienen un comportamiento. Aun así, es el término de uso más extendido en la literatura científica. (Bates, 2010; Mutshewa, 2007)

Entre las conceptualizaciones sobre qué es el comportamiento informacional articuladas por los expertos se destacan:

Taylor (1968, p. 7) lo define como "el conjunto de actividades en las cuales la información se convierte en algo útil”, en dependencia del tipo de problema que un individuo tenga que resolver.

Para Krikelas (1983, p. 11) constituye "cualquier actividad de un individuo dirigida a identificar un mensaje que satisfaga una necesidad percibida como tal".

Wilson (1981, p. 22) -uno de los principales, más destacados y prolíficos estudiosos del tema- lo definió como "la totalidad del comportamiento humano en relación con las fuentes y canales de información, incluida la búsqueda activa y pasiva de información y el uso de la información. Así, incluye la comunicación cara a cara con otros, tanto como la recepción pasiva de información (por ejemplo, al mirar los anuncios de televisión), sin intención alguna de actuar sobre la información percibida".

Parra (2006, p. 15) también sustenta la idea de que "comprende esencialmente la búsqueda de información, así como la totalidad de otros comportamientos no intencionales o pasivos, y los comportamientos intencionales que no envuelven dicha búsqueda, como el hecho de ignorar en forma persistente determinada información".

Calva (2004, p. 26) lo entiende como "la manifestación de las necesidades de información del sujeto, originadas a partir de la insuficiencia de información y conocimiento acerca de un objeto, fenómeno o acontecimiento". 
Para Fisher, Erdelez y McKechnie (2005, p. 10) representa "el área de la Documentación que se centra en investigar el modo en que los individuos necesitan, buscan, gestionan, difunden y utilizan la información en contextos diferentes".

Savolainen (2007, p. 8) coincide con esta afirmación al declarar que "es el término que describe cómo las personas necesitan, buscan, gestionan, dan y usan (la) información en diferentes contextos".

En clara alusión a la complejidad del comportamiento informacional Spink \& Coles (2006, p. 13) lo confirman como "un complejo proceso informacional y humano donde se incluye la actividad social y cotidiana de los individuos y los procesos de su vida".

Steinerová y Šušol (2007, citados por Micó, 2014, p. 14) plantean que es "la actividad humana integrada en varios niveles de procesamiento y uso de la información".

Raza et al. (2010) apunta que el comportamiento en la búsqueda de información se refiere a las estrategias para localizar información, y tiene tres componentes: personas, información y sistemas.

Bates (2010, p. 16) por su parte asevera que "es el término cuyo uso actualmente se prefiere para describir las diversas maneras en que los seres humanos interactúan con la información, en particular las maneras en que las personas buscan y utilizan la información".

Es de notar que en algunos casos los autores utilizan explícitamente el término actividad', como elemento integrante del comportamiento informacional de los seres humanos. Atendiendo al concepto de actividad humana, se tiene que este representa el contexto donde se forman y transforman los conocimientos, habilidades, hábitos, capacidades, emociones y sentimientos. De donde se desprende, entonces, que esta actividad humana tiene un componente interno y otro externo que están directamente relacionados el uno con el otro. (Figura 1)

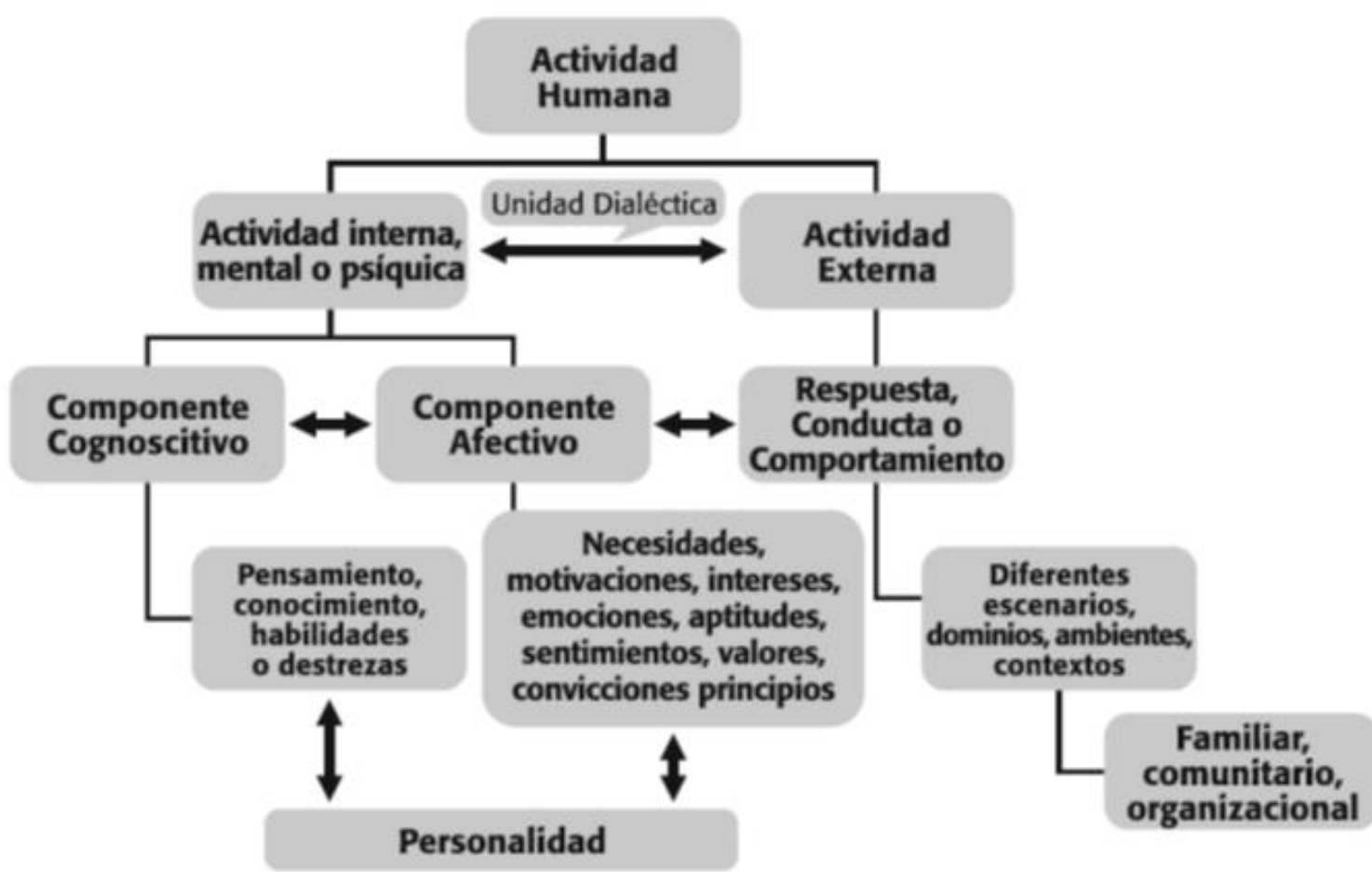

Figura 1 - Mapa conceptual de la actividad humana según el enfoque histórico-cultural (tomado de Núñez Paula \& Zayas Caballero, 2013) 
De este modo, es posible afirmar que toda actividad humana implica en sí misma un comportamiento informacional, homologando así ambos conceptos. Es decir, que toda actividad humana o toda manifestación del comportamiento humano es un comportamiento ante la información (Núñez Paula, citado por Micó, 2014), y los procesos correspondientes de selección, obtención, procesamiento, memorización, utilización y difusión de esta, que ocurren de manera consciente o inconsciente (Núñez Paula \& Zayas Caballero, 2016).

En virtud de ello, Núñez Paula \& Zayas Caballero (2013, p. 23) han propuesto una "concepción avanzada y compatible con el enfoque histórico-cultural" de comportamiento informacional humano, planteando que "es el sistema de acciones y operaciones que realizan las personas en los contextos y situaciones que actúan como mediadores, en las que se integran dialécticamente sus facetas interna o psíquica (reguladora) y externa (observable)". ${ }^{2}$

Reyes Ramírez (2011, p. 21) también señala, en este sentido, una nueva aproximación epistemológica al reconceptualizar el comportamiento informacional como un "sistema complejo de actividades sociales relacionadas con el acceso y uso de la información [con propósito innovador que evidencia los resultados de orden positivo] en la práctica social de actores competentes infoculturalmente. Se define a través de la información como atributo esencial, el valor que esta adquiere en la apropiación del conocimiento por parte de los sujetos y las implicaciones que tiene en el proceso de innovación social".

Como parte de los contextos que abarcan el comportamiento o actividad informacional humana en medio de la Sociedad de la Información y el Conocimiento imperante en la época actual, es ineludible subrayar la preponderancia y desarrollo vertiginoso de las tecnologías de la información y la comunicación, que han afectado en toda su extensión los 'saberes' y 'haceres' informativos de los individuos.

Aunque la utilización de la Teoría de la Actividad Histórico-Cultural como herramienta útil para los estudios de comportamiento informacional no ha sido totalmente explotada, sí está documentado su estimable valor y relevancia en este sentido. (Hjørland, 2002; Sundin \& Johannisson, 2005; Wilson, 2006; Uribe, 2008)

Es preciso aclarar, pues, en qué consiste el enfoque histórico-cultural (también conocido como sicología histórico-cultural) en que se fundamenta la Teoría de la Actividad y a qué se refieren concretamente ambas conceptualizaciones teóricas. El reconocido sicólogo ruso Lev S. Vygotsky fue quien dio origen a la concepción de que el desarrollo personal es una construcción cultural, que se realiza a través de la socialización con adultos de una determinada cultura, mediante la realización de actividades sociales compartidas. (Álvarez y Del Río, citado por Ibáñez Herrán, 2003)

García (2010) también ha sustentado los postulados del enfoque histórico-cultural al apuntar que para emprender cualquier investigación sobre la base de la Teoría de la Actividad se deben considerar los principios básicos de que:

- los factores sociales, culturales, históricos e institucionales juegan un importante rol en la cognición humana.

- todas las funciones mentales tienen un origen social.

- la acción (tanto social como individual) no puede ser separada del contexto social, cultural, histórico e institucional en el cual es llevada a cabo.

Para la sicología histórico-cultural el concepto de actividad tiene un protagonismo esencial, pues a través de ella se produce la creación de sentido y en ella se integran los aspectos prácticos, emocionales, relacionales y cognitivos.

Otro destacado sicólogo ruso, A. S. Leontiev, sistematizó el estudio de la actividad, dando lugar a la Teoría de la Actividad, sobre la base del principio de la sicología histórico-cultural de que la actividad intelectual no está separada de la actividad práctica. Él la definió en la siguiente manera (citado por lbáñez Herrán, 2003, p. 17):

"Las actividades se componen de acciones, que son sistemas de coordinación al servicio de fines que, a su vez, representan pasos intermedios de cara a satisfacer el motivo. Las acciones, a su vez, se componen de operaciones, o medios por los que se lleva a cabo una acción bajo condiciones específicas". (Figura 2) 


\begin{tabular}{|c|c|c|}
\hline NIVEL & ORIENTADA HACIA & RealizADA POR \\
\hline Actividad & Objetos / Motivos & Comunidad \\
\hline Acción & Objetivos & Individuos o grupos \\
\hline Operación & Condiciones & Rutinas humanas \\
\hline
\end{tabular}

Figura 2 - Jerarquía y composición de la actividad (tomado de García, 2010)

Para Leontiev, en la actividad ${ }^{3}$ el objeto con el cual interacciona el sujeto y el motivo, coinciden y se dirigen a satisfacer determinada necesidad. En las acciones, por su parte, no coinciden el objeto con el que se interacciona y el motivo. Las acciones se dirigen a objetivos que se alcanzan de forma secuencial para satisfacer la necesidad final que motiva la actividad del sujeto. En el caso de las operaciones, son las condiciones de la actividad las que determinan cómo proceder y su ejecución es automática, pudiendo darse de forma secuencial, pero también al mismo tiempo. Esto la diferencia de las acciones. (Blanco, Morales \& Rodríguez, 2010)

Esencialmente, la Teoría de la Actividad ubica a los individuos en su contexto histórico, social y cultural, que demarca e influye en la manera que estos desarrollan la suma de acciones y operaciones que componen su actividad, y en el que ejercen influencia sobre un objeto determinado a través de herramientas de mediación dentro de un conjunto de reglas que rigen la comunidad en la que los individuos actúan según los roles y/o jerarquías que poseen.

Ingwersen \& Järvelin, Wilson, y Allen et al. (citados por Roos, 2012) han resaltado el valor de la Teoría de la Actividad para el estudio efectivo, desde una perspectiva holística e interdisciplinar, del comportamiento informacional humano. Concuerdan en que brinda el marco teórico-conceptual apropiado y necesario para entender mejor el orden contextual en que las personas se desenvuelven.

Para un mayor entendimiento de la Teoría de la Actividad, Druker (2002) ha resumido las características de la actividad humana como sigue:

a) Ante una necesidad particular, el proceso activo del conocimiento produce en la mente la imagen de un objeto; por lo tanto, es objetal.

b) Siempre tiene un carácter social, ya que transcurre en activa interacción con otras personas, en comunicación y colaboración con ellas.

c) Da lugar a la formación de la conciencia; esta no se origina del conocimiento de la realidad, sino de la relación que el hombre establece con ella; es un producto subjetivo, "una forma transformadora de manifestación de aquellas relaciones, sociales por naturaleza, que se realizan en virtud de la actividad del hombre en el mundo material" (Leontiev, 1976, p. 27).

d) La actividad transformadora del hombre no es inmediata, sino que está mediatizada por signos. Antes que exista una actividad interna, mental, habrá una externa, de carácter intersicológico, que, a partir de una actividad conjunta provoca que las funciones psíquicas se interioricen, adquiriendo un carácter intrasicológico.

El análisis psíquico de la actividad y la conciencia revela sus cualidades sistémicas generales; no es correcto analizar aisladamente procesos como la memoria, la percepción o el pensamiento, sino conjuntamente y en relación con las esferas cognoscitiva y afectiva.

Tomando en cuenta la subdivisión en tres etapas o niveles que componen el comportamiento informacional (global) propuesta por Wilson (2000, p. 16), se tiene: 
- Comportamiento en la búsqueda de información (information seeking behaviour): es el propósito de buscar información conscientemente como consecuencia de la necesidad de satisfacer algún objetivo.

- Comportamiento en la recuperación de información (information searching behaviour): es un micronivel del comportamiento empleado por los individuos cuando interactúan con los sistemas de información, que incluye tanto la interacción humanocomputador (e.g.: uso del mouse, los clics, links, estrategia de búsqueda) como las acciones cognitivas (e.g.: juzgar relevancia).

- Comportamiento en el uso de la información (information use behaviour): son los actos físicos y sicológicos involucrados para incorporar la información encontrada en la existencia de la persona, en su base de conocimiento.

Después de homologar los conceptos de actividad y comportamiento (informacional), se asume que:

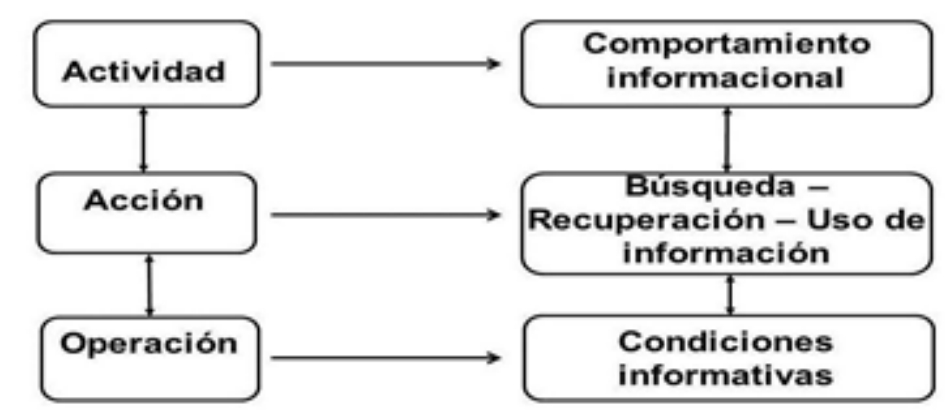

Figura 3 - Homologación conceptual entre la actividad y el comportamiento, y sus componentes (elaboración del autor)

Es necesario destacar que en esta jerarquía planteada "la actividad puede perder su motivo originario y transformarse en una acción, que realiza un tipo de relación completamente diferente respecto al mundo, otra actividad; por el contrario, una acción puede adquirir una fuerza excitadora propia y convertirse en actividad específica; finalmente, la acción puede transformarse en un procedimiento para alcanzar el objetivo, en una operación, que coadyuva a la realización de distintas acciones". (Leontiev, 1974)

Esta movilidad de los distintos "componentes" del sistema de la actividad se manifiesta, por otra parte, en el hecho de que cada uno de ellos puede fraccionarse o, por el contrario, integrar unidades que anteriormente eran independientes. (Núñez Paula, 2004)

Ingwersen (2004, citado por Tramullas, 2013) también ha dejado claro la naturaleza dinámica y cambiable de los procesos de búsqueda de información, al remarcar que "la búsqueda de información ha sido comprendida como un proceso en el cual la comprensión del usuario de sus tareas o problemas, necesidades de información, relevancia, criterios y del espacio de información disponible, evoluciona..."

En el estudio del comportamiento informacional los dos paradigmas prevalecientes son el centrado en el sistema (llamado también tradicional) y el centrado en el usuario (llamado también alternativo). Cada uno de ellos responde a corrientes de pensamiento diferentes: el primero al conductismo, y el segundo al cognoscitivismo. Sus características fundamentales se resumen en (Dervin \& Nilan, 1986):

Paradigma orientado al sistema o tradicional:

- Información objetiva

- Usuarios pasivos 
- Transituacional

- Visión parcial del proceso de búsqueda de información

- Conducta externa

- Individualidad caótica

- Metodología cuantitativa

Paradigma orientado al usuario o alternativo:

- Información subjetiva

- Usuarios activos

- Situacional

- Visión integral del proceso de búsqueda de información

- Pensamientos internos

- Individualidad sistemática

- Metodología cualitativa

Estas visiones paradigmáticas deben estar conjugadas entre sí e integradas congruentemente al análisis comportamental que se emprenda para alcanzar una eficaz comprensión global y la efectividad deseada.

En los marcos teórico-metodológicos y conceptuales de estos paradigmas se inscriben varios modelos de análisis que intentan esquematizar los modos o maneras en que las personas se comportan informacionalmente. Entre ellos sobresalen los esbozados por Wilson (1981, 1996, 1999, 2006) -quien igualmente defiende la viabilidad y utilidad de los principios de la Teoría de la Actividad parauna mejor comprensión de los procesos que promueven y tienen lugar en el comportamiento informacional humano-,Belkin (Anomalous State of Knowledge - ASK, 1980), Krikelas (1983), Dervin (Sense-making, 1983), Taylor (User-values, 1986), Kuhlthau (Information Seeking Process, 1989), Ellis (1989), Ellis, Cox \& Hall (1993), Leckie, Pettigrew, Sylvain \& Ingwersen (1996), Niedźwiedzka (2003), Choo (2003) y Godbold (2006).

No se puede dejar de aludir a una nueva y emergente perspectiva de estudio del comportamiento informacional: las prácticas informacionales, que representan un estalón superior en este sentido y cuyo interés principal radica (y roza justamente con los planteamientos de la Teoría de la Actividad) en esbozar la 'vida informacional' de las personas a partir de un enfoque construccionista y sociocultural, en el que se imbrican e implican todas las facetas y esferas de acción y actuación de los individuos. (Bogliolo Sirihal; Ávila Araújo; Anastásio de Paula, 2017)

Preciso es aclarar que la Teoría de la Actividad no pretende suplantar los modelos o perspectivas de estudio del comportamiento informacional establecidos previamente por la comunidad científica, sino más bien procura aportar razones y elementos que impliquen distinguir y reconocer fenómenos de la realidad que no han sido (suficientemente) estudiados. De modo que se confirma la riqueza y complejidad del campo de 'lo real', que se presenta como un desafío permanente para la investigación científica. Este hecho se hace todavía más evidente y preponderante en los estudios de comportamiento informacional, los cuales tratan con objetos que son también sujetos (usuarios) que utilizan dispositivos en constante cambio (técnicas y tecnologías informacionales) para lidiar con algo de naturaleza tan fluida y cambiante como es la información. (Ávila Araújo, 2016)

Barros, Vélez \& Verdejo (2004) han subrayado que la Teoría de la Actividad Histórico-Cultural constituye un marco teórico-metodológico funcional para analizar desde una combinación de perspectivas objetivas, ecológicas y socioculturales la actividad humana. 
Nardi (1996) también ha sugerido la Teoría de la Actividad Histórico-Cultural como uno de los presupuestos teóricos que mejor describe los procesos de la actividad humana, pues ofrece amplias posibilidades de análisis y flexibilidad para establecer conclusiones teóricas, a la vez que representa una teoría que se desarrolla y progresa con la expansión de sus postulados a las más diversas áreas de conocimiento.

Adams et al. (citado por García, 2010) resume las características de la Teoría de la Actividad Histórico-Cultural en estos elementos:

- jerarquía de las actividades (basado en el nivel de actividades especificado por Leontiev: actividad, acción y operación)

- carácter colectivo de las actividades (la dirección hacia un objetivo involucra habitualmente a varias personas trabajando como comunidad)

- carácter contextual de las actividades (las actividades no se llevan a cabo en el vacío, siempre hay un contexto que las condiciona con sus circunstancias)

- dinamismo de las actividades (las actividades no son estáticas y para comprenderlas es necesario el análisis histórico de su evolución)

- la mediación (la actividad está mediada por herramientas, reglas y división de tareas)

- elección contextual de las acciones (repertorio de acciones y operaciones que son creadas para hacer posible una actividad cualquiera)

- comprensión de las acciones en su contexto (las acciones no pueden ser comprendidas aisladamente sino en el marco de la actividad de la que forman parte; por lo tanto, para un desempeño eficiente de la acción es necesario comprender el objeto de la actividad)

- $\quad$ planificación del trabajo (la actividad puede ser modificada en el proceso de su ejecución conforme a las condiciones del contexto, por lo que un plan no impone una prescripción)

- el valor de las excepciones (las desviaciones de un plan original pueden ocurrir, y dan oportunidad para el aprendizaje que genera otras formas de ejecución)

- granularidad basada en la perspectiva (puede suceder que una actividad o acción dependa de la perspectiva particular de quien la ejecute)

El desarrollo de la Teoría de la Actividad Histórico-Cultural partió de considerar primeramente la actividad como la interacción de sujeto y objeto a través de artefactos de mediación (Vygotsky, 1933), luego definir la estructura de la actividad, compuesta por acciones y operaciones (Leontiev, 1984), hasta incluir más recientemente el contexto en el que se encuentran inmersos sujeto, objeto y artefactos mediadores, lo que se manifiesta en los sistemas de producción, distribución, consumo e intercambio de una comunidad y materializados por las reglas, la división del trabajo y las interacciones de todos los miembros de la comunidad por alcanzar el objetivo del sistema y transformar el objeto (Engeström, 2000). De este modo, la "actividad mediada" de Vygotsky y Leontiev se tornó en "sistemas de actividad" por Engeström.

Estos sistemas de actividad tienen la estructura siguiente: 


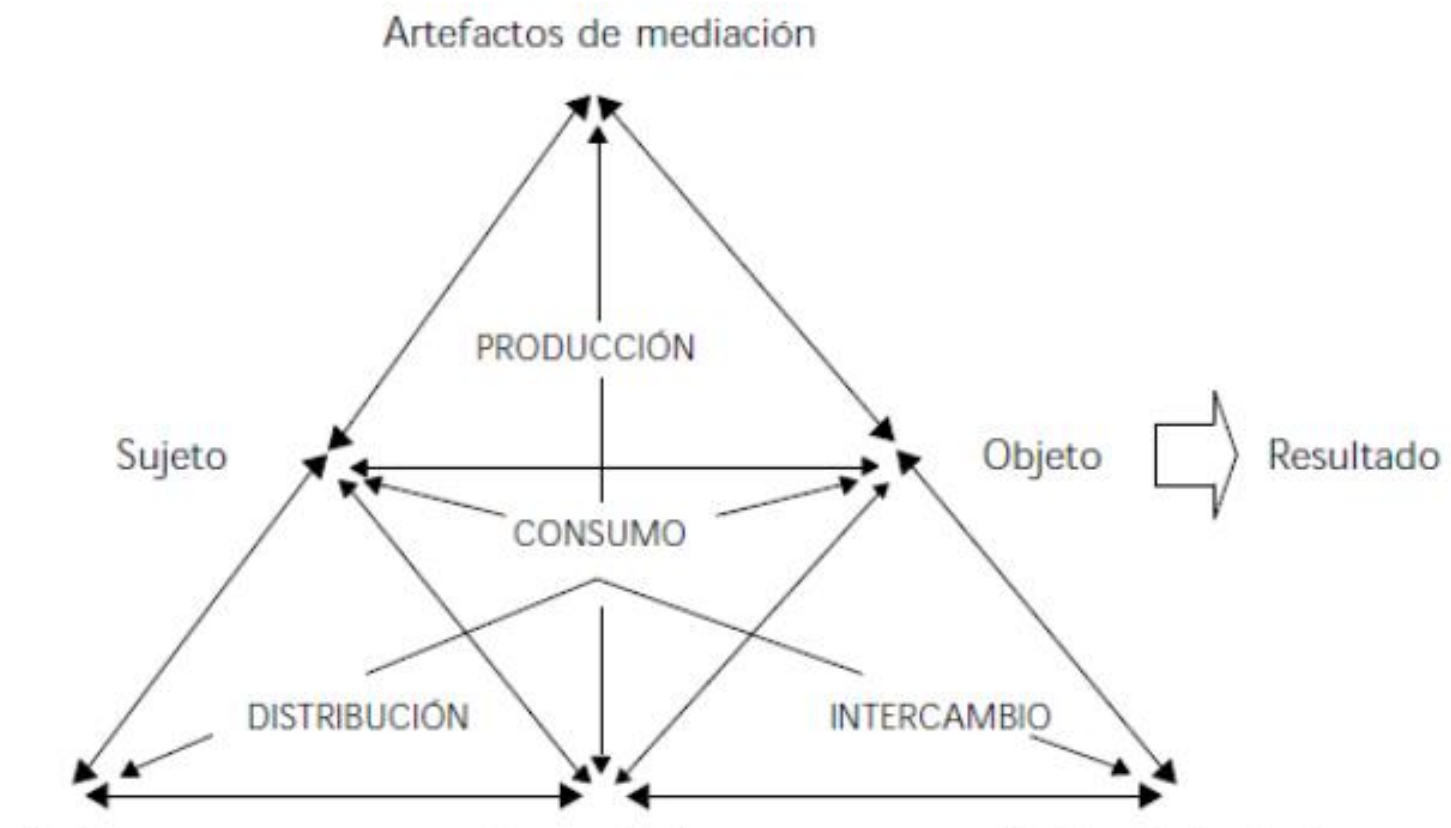

Reglas

Comunidad

División del trabajo

Figura 4 - Estructura sistémica de la actividad según Engeström (tomado de Engeström, 2000)

Donde cada uno de los elementos intervinientes representa:

- Sujeto4: individuo o subgrupo cuya agencia se toma como el punto de vista del análisis.

- Objeto: espacio de problema o parte del mundo material al que está dirigida la actividad en función de alcanzar el resultado requerido, y que demanda ser transformado.

- Herramientas: artefactos mediadores, sean 'técnicos' o 'psicológicos'.

- Comunidad: colectivo de individuos y grupos cuya actividad está orientada hacia el objeto compartido.

- División del trabajo: división horizontal de actividades y división vertical de poder y responsabilidad; quién realiza qué acciones en relación al objeto compartido.

- Reglas: cultura compartida del sistema de actividades con regulaciones explícitas e implícitas, restricciones, procesos, prácticas culturales, normas, puntos de vista, convenciones, etcétera.

- Resultado ('outcome' o salida): aquello que se espera alcanzar o lograr.

Cada uno de estos componentes representan los actores y factores que demarcan el espacio contextual de participación en que tiene lugar la actividad o comportamiento informacional individual y/o colectivo.

En este sistema de actividades, la relación sujeto-objeto está mediada por los artefactos mediadores o herramientas ${ }^{5}$, que pueden ser físicos, intelectuales o materiales (e.g.: programas, instrumentos); la relación sujeto-comunidad está mediada por las reglas; la relación (e.g.: leyes); la relación objeto/comunidad está mediada por la división del trabajo. Cada uno de estos términos es históricamente formado y susceptible al cambio (Kuutti, 1995, citado por Roos, 2012). 
Reafirmando esta idea, Núñez Paula (2004) declara que producto a la naturaleza social del hombre, cuyas relaciones se basan en el lenguaje, en la conciencia y en la transformación consciente de la realidad, la relación sujeto-objeto ocurre mediatizada por -e integrada cognoscitiva y afectivamente a- la historia de sus relaciones con otras personas o grupos y con su contexto organizacional y social.

Otros conceptos centrales a considerar, en el intento de analizar el comportamiento informacional desde la Teoría de la Actividad Histórico-Cultural, son los motivos y las necesidades informativas. Toda actividad humana está impulsada y dirigida por un motivo (rector o complementario), que a su vez está asociado a una necesidad por alcanzar una meta u objetivo. (Núñez Paula, 2004)

Leontiev (1978) planteó que la actividad es "el proceso originado y dirigido por un motivo, dentro del cual ha tomado forma de objeto determinada necesidad".

Los motivos, de acuerdo a su alcance funcional en la regulación de la actividad o comportamiento se pueden clasificar, según González Rey (1989), en:

1. De carácter objetal: aquellos objetos, situaciones o resultados concretos que satisfacen una necesidad.

2. Presentes ante condiciones actuantes sobre el sujeto: Cuando lo que motiva al individuo son valoraciones, reconocimientos, placeres estéticos, situaciones organizativas, ventajas económicas, y otras condiciones.

3. Orientadores de la actividad y de las relaciones del hombre: Son los motivos que se interiorizan por la persona durante la vida, como sistemas de reglas elementales de comportamiento ante determinadas situaciones y que, al interiorizarse o automatizarse, se salen parcial o totalmente de la conciencia, sin embargo, regulan el comportamiento del sujeto en las situaciones actuantes concretas.

4. Orientadores del sentido de la vida: Son los que tienen un carácter más abstracto y consciente, como las valoraciones, las convicciones, las actitudes, que regulan fundamentalmente el comportamiento del sujeto en la situación presente, pero bajo el control interactivo de la reflexión sobre el sentido del comportamiento para la vida.

5. Tendencias orientadoras de la personalidad: orientan el comportamiento hacia sus objetivos esenciales en la vida. Tienen un sentido personal y consciente para el sujeto, tales como los ideales (la visión, en el caso de las organizaciones), las intenciones, la autovaloración, y otras situaciones. Implican una proyección hacia el futuro, consciente y bien estructurada.

Muy estrechamente ligado a los motivos están las necesidades de información, que actúan como trasfondo de los primeros. Según Wilson (1981) "se constituyen a partir de otras necesidades originadas en los diferentes contextos de la experiencia y la acción; a partir del contexto físico y biológico y de los contextos de trabajo, sociales, políticos, etcétera".

Según Salazar Hernández (1993) las necesidades están condicionadas por:

- El nivel de experiencia sobre la disciplina de interés.

- El tiempo de experiencia en la disciplina.

- La actividad individual o grupal del usuario.

- Su persistencia.

- Su motivación. 
- Su manejo del lenguaje.

- Su capacidad de análisis de la información.

- La disponibilidad de los recursos de información.

- El medio en el que se desarrolla el usuario: situación social, política y económica de su entorno.

Las contradicciones son otro elemento fundamental en la Teoría de la Actividad Histórico-Cultural, y se manifiestan a través de tensiones estructurales acumuladas históricamente. Pueden estar dadas, por ejemplo, por la introducción de una nueva tecnología en un sistema determinado, y no solo producen perturbaciones y conflictos sino también conducen al surgimiento de soluciones innovadoras para resolverlas y cambiar la actividad. (Engeström, 2001)

Gregory Z. Bedny es otro autor que ha abordado con profundidad la Teoría de la Actividad Histórico-Cultural ${ }^{6}$. Añadió al modelo de sistemas de actividades de Engeström el elemento de la retroalimentación que se recibe luego de que los individuos obtienen los resultados de su actividad e introducen nuevos cambios al 'producto' alcanzado. (Figura 5)

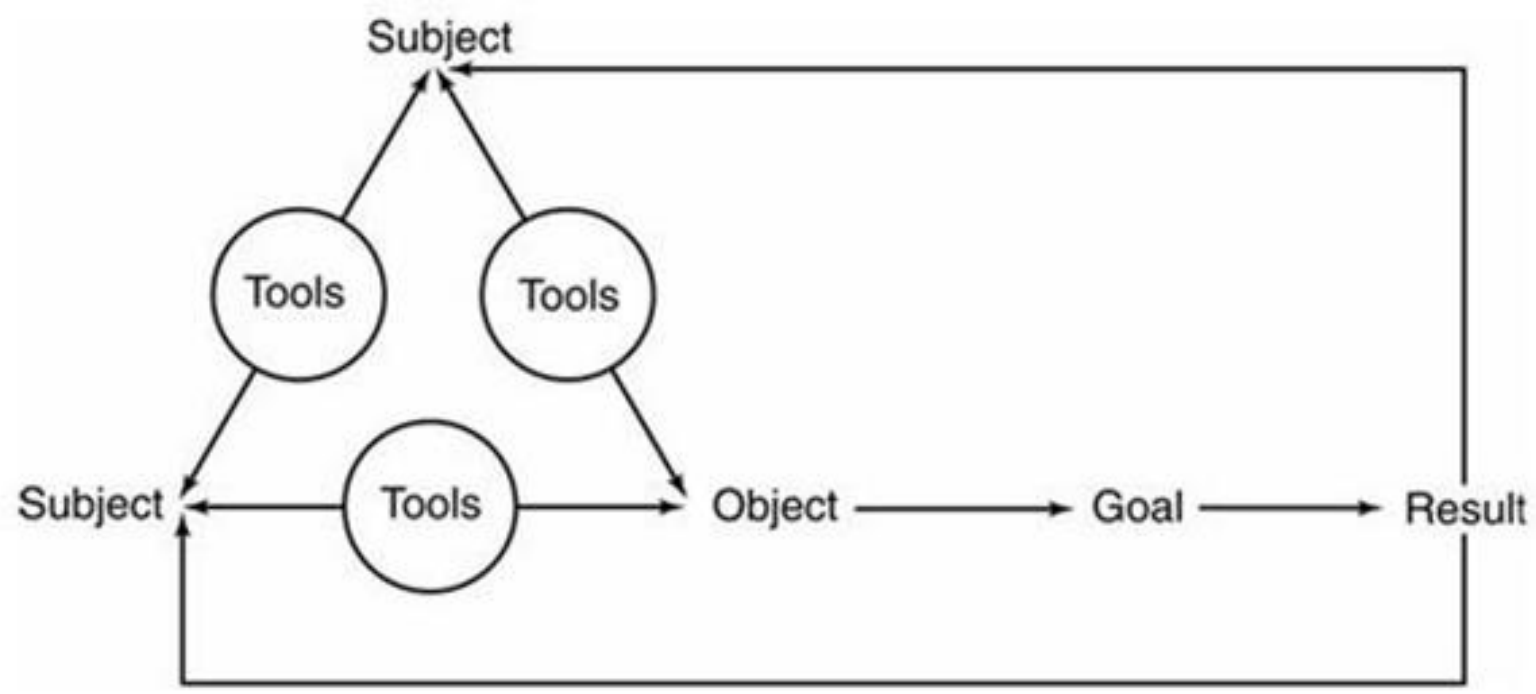

Figura 5 - Esbozo de la Teoría Sistémico-Estructural de la Actividad según Bedny (tomado de Bedny, 2003)

Este autor conceptualiza la actividad como "un sistema orientado a una meta, donde la cognición, el comportamiento y la motivación están integrados por un mecanismo de autorregulación para alcanzar una meta consciente". Ademas clasifica la actividad en dos tipos: 'objetiva' (orientada al objeto) y 'subjetiva' (orientada al sujeto). En la primera, el sujeto modifica al objeto a través de las herramientas o instrumentos, y en la segunda, también conocida como interacción social, involucra a dos o más sujetos que comparten información ${ }^{7}$ y sostienen contactos interpersonales y de entendimiento mutuo. (Bedny \& Harris, 2004)

Mwanza (2002) ha propuesto el siguiente modelo de ocho pasos para el análisis de la Teoría de la Actividad: 


\begin{tabular}{|l|l|l|}
\hline \multicolumn{1}{|c|}{ Identifica: } & Preguntas a formular: \\
\hline Paso 1 & La Actividad de Interés & ¿En qué clase de actividad estoy interesado? \\
\hline Paso 2 & Objetivo & ¿Por qué tiene lugar la actividad? \\
\hline Paso 3 & Sujetos & ¿Quién está involucrado en llevar a cabo esta actividad? \\
\hline Paso 4 & Herramientas & $\begin{array}{l}\text { ¿Qué significado tiene para los sujetos desempeñar esta } \\
\text { actividad? }\end{array}$ \\
\hline Paso 5 & Reglas \& Regulaciones & $\begin{array}{l}\text { ¿Hay algunas normas, reglas o regulaciones gobernando el } \\
\text { desempeño de esta actividad? }\end{array}$ \\
\hline Paso 6 & División del Trabajo & $\begin{array}{l}\text { ¿Quién es responsable de que se lleve a cabo esta actividad, } \\
\text { y cómo están organizados los roles? }\end{array}$ \\
\hline Paso 7 & Comunidad & $\begin{array}{l}\text { ¿Cuál es el ambiente en el que esta actividad es llevada a } \\
\text { cabo? }\end{array}$ \\
\hline Paso 8 & Resultado & $\begin{array}{l}\text { ¿Cuál es el Resultado deseado para llevar a cabo esta } \\
\text { actividad? }\end{array}$ \\
\hline
\end{tabular}

Figura 6 - Modelo de Ocho Pasos para el Análisis Metodológico de la Teoría de la Actividad (Mwanza, 2008, tomado de García, 2010)

Dando respuestas a estas interrogantes se tendrá claridad de los elementos componentes que intervienen en una actividad determinada. Siguiendo este modelo se pueden analizar los subsistemas de actividades, de manera que se facilita el estudio del fenómeno.

Atendiendo a lo anterior se tiene que la actividad o comportamiento informacional representa un sistema general que engloba a los subsistemas de búsqueda, recuperación y uso de información -vistos anteriormente por Wilson-.

Para descubrir el comportamiento informacional desde la perspectiva de la Teoría de la Actividad HistóricoCultural es preciso aplicar estos presupuestos, tomando como unidad básica de análisis a la actividad de los individuos (ya sea a nivel personal o grupal).

Para su eficiente aplicación se debe tomar en cuenta que, antes que una metodología, constituye un modelo para el análisis a través de una aproximación transdisciplinar, ofreciendo herramientas conceptuales y principios metodológicos que deben ser adaptados según la naturaleza concreta de aquello que es objeto de escrutinio. (Engeström, 1993)

\section{Consideraciones finales}

El comportamiento informacional humano continúa siendo objeto de estudio como instrumento revelador de los procederes informativos de los individuos en tanto gestionan la información que necesitan.

Ha habido una evolución de la época en la que solo se atendía a cuestiones meramente cuantitativas en los estudios de usuarios, no despreciables pero insuficientes para describir/discernir la complejidad de los múltiples entornos que median en los procesos de búsqueda informativa, se da un salto cualitativo hacia enfoques inter- $y$ transdisciplinarios que aporten luz más profundamente en el esclarecimiento de estos temas.

Es en esta dirección que destaca la Teoría de la Actividad Histórico-Cultural, subproducto de la sicología histórico-cultural, proponiendo un marco de principios teórico-metodológicos aplicables a los estudios de comportamiento informacional (que implica las etapas o procesos de búsqueda, recuperación y uso de información) en tanto consideran la actividad humana -que es inherentemente informacional- como la unidad mínima de análisis de los individuos como seres sociales. (De ahí que el comportamiento es visto como sinónimo de la categoría sicológica de la actividad, lo cual conlleva un componente interno o síquico y otro externo o conductual)

Los contextos (socioeconómicos e histórico-culturales), la jerarquía de actividades (acciones, operaciones), la historicidad y las contradicciones en los sistemas de atividades (que involucran las relaciones de producción, consumo, reparto e intercambio [de información] entre sujeto-objeto a través de herramientas de mediación como el lenguaje y los símbolos/signos- dentro de una comunidad específica, regida por determinadas leyes o estatutos, y con una distribución del trabajo, tareas o funciones establecidas) constituyen ejes cardinales que (re)direccionan las motivaciones y necesidadesinformativas de los sujetos. 
A partir de estas nuevas visiones teórico-conceptuales en la literatura especializada se ha ampliado y equiparadola idea del comportamiento informacional a todas las actividades relacionadas con el acceso y uso de la información de las personas en medio de su contexto histórico-social.

Es decir, desde el enfoque histórico-cultural -de origen sicológico- y la perspectiva de la Teoría de la Actividad, el comportamiento informacional es mejor comprendido como "el sistema de acciones y operaciones que realizan las personas en los contextos y situaciones que actúan como mediadores, en las que se integran dialécticamente sus facetas interna o síquica [reguladora] y externa [observable]" (Núñez Paula \& Zayas Caballero, 2013), lo cual clarifica y adecua más eficientemente la estructura de análisis, estudio e interpretación global del fenómeno de los procesos del comportamiento informacional humano (tanto en su nivel individual como grupal u organizacional).

\section{Referencias}

ÁVILA ARAÚJO, C. A. (2016). Estudos de usuários da informação: comparação entre estudos de uso, de comportamento e de práticas a partir de uma pesquisa empírica. [en línea] Informação em Pauta. Fortaleza (Brasil), 1 (1): 61-78. Disponible en: http://www. periodicos.ufc.br/index.php/informacaoempauta/article/view/2970/2694.pdf Consultado: 14 de marzo, 2017.

BÁRZAGAS, Y. (2005). Alfabetización y comportamiento informacional. Estudio de caso. Tesis (Licenciado en Bibliotecología y Ciencias de la Información). La Habana: Universidad de La Habana, 81 h.

BATES, M. J. (2010). Information behaviour. University of California - Los Angeles. Disponible en: http://pages.gseis.ucla.edu/faculty/bates/articles/information-behavior.html Consultado: 9 de abril, 2013

BEDNY, G.; KARWOWSKI, W. (2007). A systemic-structural theory of activity. Applications to human performance and work design. Boca Raton, FL: CRC Press.

BOGLIOLO SIRIHAL DUARTE, A.; ÁVILA ARAÚJO, C. A.; ANASTÁSIO DE PAULA, C. P. (2017). Práticas informacionais: desafios teóricos e empíricos de pesquisa. [en línea] Informação em Pauta. Fortaleza (Brasil), 1 (1): 56-66. Disponible en: http://periodicos.ufc.br/informacaoempauta/article/view/20650/31077 Consultado: 14 de marzo, 2017

CASE, D. (2002). Looking for Information: a Survey of Research on Information Seeking, Needs and Behaviour. San Diego: Academic Press, 2002.

DERVIN, B.; NILAN, M. (1986). Information needs and uses. Annual Review of Information Science and Technology, v. 48, n. 21: 3-33.

DRUKER, A. V. (2002). Aplicación del Enfoque Histórico-cultural y la Teoría de la actividad al Sistema Didáctico para la enseñanza de la asignatura Materiales y Procesos. (Tesis de Maestría) Universidad Nacional de Misiones, Argentina. 164 h.

ENGESTRÖM, Y. (2001). Expansive learning at work: toward an activity theoretical reconceptualization. Journal of Education and Work, v. 14, n. 1: 133-156.

FISHER, K.; JULIEN, H. (2009). Information behavior. Annual Review of Information Science \& Technology, v. 43, n. 9: 317358.

GONZÁLEZ-TERUEL, A. (2011). La perspectiva del usuario y del sistema en la investigación sobre el comportamiento informacional. Teoría de la Educación. Educación y Cultura en la Sociedad de la Información, v. 12, n. 1: 9-27.

KUHLTHAU, C. (1991). Inside the search process: information seeking from the user's perspective. Journal of the American Society for Information Science, v. 42, n. 5: 361-371.

MARTÍNEZ COMECHE, J. A.; HORCAS PULIDO, J.; PIÑA LANDABURU, Z. (2014). Análisis de procesos cognitivos en el comportamiento de estudiantes de último curso de bachillerato y primer curso de carrera involucrados en tareas de clasificación, cálculo, memorización y categorización de información. Investigación Bibliotecológica, v. 28, n. 64.

MCKECHNIE, L.; PETTIGREW, K.; JOYCE, S. (2001). The origins and contextual use of theory in human information behaviour research. New Review of Information Behaviour Research, v. 14, n. 2: 47-63.

MICÓ MENDOZA, M. (2014). Factores que influyen en el comportamiento informacional de estudiantes de 4to. año de Ciencias de la Información en la gestión de información personal en Facebook (Máster en Ciencias de la Información). La Habana: Universidad de La Habana, Facultad de Comunicación, 120 h.

NÚÑEZ PAULA, I.; ZAYAS CABALLERO, I. (2013). Perspectiva histórica y metodológica del sistema conceptual relativo al comportamiento informacional. Psicología Pedagógica, v. 8-9: 50-75. 
(2016). Análisis de modelos sobre Comportamiento Informacional, desde un enfoque sociosicológico. Bibliotecas: Anales de Investigación, v. 12, n. 1: 63-89.

RODRÍGUEZ PINTÓN, I. (2010). Comportamiento informacional de los profesionales de la prensa cubana. Estudio aplicado al proceso de acceso y uso de la información. (Licenciado en Bibliotecología y Ciencias de la Información). La Habana: Universidad de La Habana. $111 \mathrm{~h}$.

ROOS, A. (2012). Activity theory as a theoretical framework in the study of information practices in molecular medicine. Information Research, v. 17, n. 32: paper 526. Disponible en: http://InformationR. net/ir/17-3/paper526.html Consultado: 15 de septiembre, 2016.

SÁNCHEZ DÍAZ, M. (2010). Competencias informacionales en la formación de las BioCiencias en Cuba. (Tesis Doctoral). Granada (España): Universidad de Granada, 174 h.

SAVOLAINEN, R. (2005). Everyday life information seeking. En: Fisher, K. E., Erdelez, S. \& McKechnie, L. (Eds). Theories of Information Behaviour, Information Today, Medford: ASIST Monograph Series, 143-148.

SPASSER, M. A. (1999). Informing information science: the case for activity theory. Journal of the American Society for Information Science, v. 50, n. 12: 1136-1138.

TRAMULLAS, J. (2013). Sigue al conejo blanco: misterios, usuarios y comportamiento informacional. Disponible en: http://es.slideshare.net/tramullas/upf-info-behverb Consultado: 23 de marzo, 2015.

URIBE TIRADO, A. (2008). Diseño, implementación y evaluación de una propuesta normativa en alfabetización informacional mediante un ambiente virtual de aprendizaje a nivel universitario. Caso Escuela Interamericana de Bibliotecología, Universidad de Antioquia. (Máster en Ciencias de la Documentación), Medellín (Colombia): Universidad EAFIT, 535 h.

VALERO RIVERO, D.; PONJUÁN DANTE, G. (2014). Análisis del comportamiento informacional en la comunidad científica de la provincia de Sancti Spíritus formada a partir de un proyecto colaborativo. [En línea] Revista Cubana de Información en Ciencias de la Salud, v. 25, n. 2: 183-198 Disponible en: http://acimed.sld.cu/index.php/acimed/article/view/574/401 Consultado: 13 de junio, 2014

WILSON, T. D. (1981). On user studies and information needs. Journal of Documentation, v. 37, n. 3: 3-15.

(2000). Human Information Behaviour. Informing Science, v. 3, n. 2: 49-55.

ZAYAS CABALLERO, I. (2012). Comportamiento Informacional: Aproximaciones teóricas. (Licenciado en Bibliotecología y Ciencias de la Información). La Habana: Universidad de La Habana, $100 \mathrm{~h}$. 


\section{Datos del autor}

\section{Raciel García Rodríguez}

Profesor de la Universidad de Matanzas (Cuba). Dirección de Informatización/Gestión de Información y el Conocimiento. Licenciatura/Maestría en Bibliotecología y Ciencias de la Información por la Universidad de La Habana (Cuba).

raciel.garcia@umcc.cu

Recibido - Received: 2016-07-12

Aceptado - Accepted: 2017-03-19

'Del latín activitas, activas = actuar. Mediatiza la vinculación del sujeto con el mundo real, y es generadora del reflejo psíquico, el cual, a su vez, mediatiza a la propia actividad.

${ }^{2}$ La actividad interna y la externa son directamente proporcionales, y así también los correspondientes procesos (transformadores) de internalización y externalización se producen en paralelo, donde el sujeto y el objeto son afectados mutuamente.

${ }^{3}$ Vale destacar que las habilidades se relacionan con la actividad consciente y los hábitos con la actividad inconsciente, o sea, realizada de forma automática. Ambos representan altos niveles de dominio de la ejecución de que se trate, pero constituyen dos niveles diferentes de ejecución de la actividad, la habilidad a un nivel más razonado o consciente y la operación de una forma más automática y en ese sentido inconsciente. (Entiéndase que el uso de los términos 'consciente' e 'inconsciente' no tiene relación con el estado de vigilia). Psicólogos como el propio A. N. Leontiev, S. L. Rubinstein y L. S. Vygotsky consideraron como formas de asimilación de la actividad los conceptos de hábitos, habilidades y capacidades, los cuales reflejan el dominio de las operaciones, acciones y actividad, respectivamente. (Bermúdez \& Rodríguez, 2003)

${ }^{4}$ Roos (2012) sugiere que, desde el punto de vista de la Teoría de la Actividad y considerando que las actividades son conscientes y dinámicas, y son propulsadas por motivos -sean estos globales o específicos-, también se le denomine actor, pues así se toma en cuenta la naturaleza interactiva del proceso donde el actor transforma las metas/objetivos en salidas/resultados de una mejor forma -más protagónica, participativa y decisiva- que el usuario.

${ }^{5}$ También se incluyen dentro de estos los signos (e.g.: el lenguaje), símbolos o imágenes (que implican significados).

${ }^{6}$ Para este autor denominada Teoría Sistémico-Estructural de la Actividad.

${ }^{7} \mathrm{~A}$ este fenómeno los expertos en estudios de comportamiento informacional en la literatura especializada se refieren como information sharing o comportamiento colaborativo.

\section{$((c))$ EY}

This work is licensed under a Creative Commons Attribution 4.0

United States License.

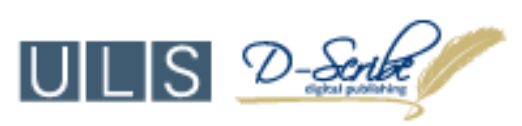

This journal is published by the University Library System of the University of Pittsburgh as part of its D-Scribe Digital Publishing Program and is cosponsored by the University of Pittsburgh Press. 